\title{
Diagnostic Performance of Xpert MTB /RIF in Comparison with LED Fluorescence Microscopy and Culture in Suspected Cases of Pulmonary Tuberculosis
}

\author{
Vishnu Prasad Shenoy ${ }^{D}$, Ajay Kumar and Kiran Chawla* \\ Department of Microbiology, Kasturba Medical College Manipal, Manipal Academy of Higher Education, \\ Karnataka, India.
}

\begin{abstract}
GeneXpert MTB/RIF assay (GeneXpert) is relatively new technology in India and it is important to generate data on its efficacy for diagnosis of Tuberculosis. Thus; present study was undertaken to compare the diagnostic performance of GeneXpert for diagnosis of pulmonary tuberculosis (PTB) in comparison with Auramine $O$ staining based Light Emitting Diode- Fluorescence Microscopy (LEDFM) considering culture as gold standard. A total of 662 (451 BAL, 211 Sputum) respiratory samples obtained from patients suspected of PTB were tested for smear microscopy and GeneXpert assay and were compared with culture results. Rifampicin resistance was compared with Genotype MTBDR plus assay. Sensitivity, specificity, positive predictive value (PPV) and negative predictive values (NPV) of the assays were calculated using SPSS version 22 . On comparing with culture the sensitivity, specificity,

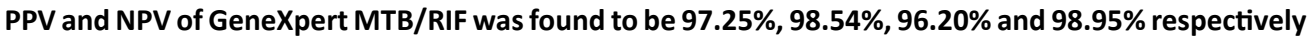
whereas sensitivity, specificity, PPV and NPV of smear microscopy was found to be $73.08 \%, 100 \%, 100 \%$ and $\mathbf{9 0 . 7 4 \%}$ respectively. Rifampicin resistance was detected in 11 (1.66\%) samples by GeneXpert. Five $(0.75 \%)$ samples among them were found to be MDR whereas one sample showed false positive result for rifampicin resistant when compared by Genotype MTBDR plus assay. GeneXpert MTB/RIF assay offers much higher sensitivity as compared to Auramine $O$ staining based LED-FM and seems to be a promising tool for TB diagnosis. Further longitudinal studies are required to evaluate the potential of this assay at primary health care settings and diagnosis of Extrapulmonary TB.
\end{abstract}

Keywords: Culture, Diagnosis, GeneXpert, Microscopy, Mycobacterium tuberculosis.

*Correspondence: kiran.chawla@manipal.edu

(Received: 28 July 2019; accepted: 02 September 2019)

Citation: Vishnu Prasad Shenoy, Ajay Kumar and Kiran Chawla, Diagnostic Performance of Xpert MTB /RIF in comparison with LED Fluorescence Microscopy and Culture in Suspected Cases of Pulmonary Tuberculosis, J Pure Appl Microbiol., 2019; 13(3): 1461-1465. https://doi.org/10.22207/JPAM.13.3.16

(c) The Author(s) 2019. Open Access. This article is distributed under the terms of the Creative Commons Attribution 4.0 International License which permits unrestricted use, sharing, distribution, and reproduction in any medium, provided you give appropriate credit to the original author(s) and the source, provide a link to the Creative Commons license, and indicate if changes were made. 


\section{INTRODUCTION}

Tuberculosis (TB) remains a major health care issue in India. India accounted for highest burden for TB (2.74 million) and multi drug resistant TB in 2017, which accounted for 4.1 lakhs lives ${ }^{1}$. Though TB is curable with appropriate treatment such huge number of deaths signifies the need of early accurate diagnosis, prompt treatment and proper monitoring of such cases. Over years lack of inadequate tools for rapid diagnosis of TB remained major obstacle in TB control programmes especially in resource poor countries like India. For diagnosis of TB, Mycobacterial culture is the gold standard but it takes 6-8 weeks to obtain final result and requires sophisticated laboratory facility and technically skilled person to perform the test ${ }^{2,3}$. Thus, majority of TB control programmes were based on Ziehl-Neelsen (ZN) staining based smear microscopy which has suboptimal sensitivity ${ }^{4}$. In 2011, WHO endorsed auramine based light-emitting diode fluorescent microscopy (LED-FM) for national tuberculosis control programmes in developing countries ${ }^{5}$. Auramine-LED-FM has shown increased sensitivity as compared to conventional Ziehl-Neelsen microscopy under programmatic conditions in India ${ }^{6}$. Recently WHO endorsed GeneXpert (Xpert ${ }^{\circledR}$ MTB/RIF assay); a fully automated cartridge based nucleic acid amplification (CB-NAAT) assay which simultaneously detects Mycobacterium tuberculosis and most common genetic mutations associated with Rifampicin resistance with a turnaround time of less than 2 hours ${ }^{7}$. Preliminary studies assessing the performance of GeneXpert have reported very high sensitivity and specificity in Pulmonary TB (PTB) cases $^{8-10}$. GeneXpert is relatively new technology in India and it is important to generate data on utility of this assay for diagnosis of TB. Thus, present study was undertaken to evaluate the diagnostic efficacy of GeneXpert (Cepheid, US) assay for diagnosis of PTB in comparison with Auramine $O$ staining based LED-FM.

\section{MATERIALS AND METHODS Study setting}

Present research was carried out in Department of Microbiology at a tertiary care centre in Karnataka, India from August 2016 to August 2017 after obtaining approval from the
Institutional Research and Ethics Committee. A total of 662 (451 BAL, 211 Sputum) non repetitive samples obtained from patients suspected of pulmonary TB were examined by Auramine $O$ staining based LED-FM and GeneXpert. All samples were divided into two equal aliquots; one aliquot was used for GeneXpert whereas other aliquot was used for Auramine $\mathrm{O}$ staining and Culture.

\section{Smear Microscopy}

For all samples AFB smears were prepared and rest of samples were stored at $4^{\circ} \mathrm{C}$ to use later for validation of GeneXpert and smear microscopy results. The smears were stained with $0.3 \%$ Auramine phenol $O$ for 10 minutes and then were decolourised with $1 \%$ acid alcohol solution for 2 minutes and finally counter stained with $0.1 \%$ potassium permanganate for 1 minute. All stained smears were examined under LED-FM following the guidelines of Revised National Tuberculosis Control Program (RNTCP) ${ }^{11}$.

\section{GeneXpert Assay}

GeneXpert assay was performed following the instructions given in product insert provided by manufacturer along with the kit. Briefly untreated sample was mixed with sample reagent at a ratio of $1: 2$ and the contents were mixed manually by gentle agitation and sample reagent mixture was kept at room temperature for 15 minutes and $2 \mathrm{ml}$ of mixture was transferred to the GeneXpert cartridge. Then cartridge was loaded into the GeneXpert device and finally the results were read after completion of the test. All samples that showed invalid results were repeated.

\section{Validation of results}

All samples were further processed using the NALC-NaOH ( $\mathrm{N}$-acetyl-L cysteine- sodium hydroxide) method and were inoculated in Mycobacterial Growth Indicator Tubes (MGIT) or Lowenstein Jensen (LJ) medium and were monitored for positive growth till 6- 8 weeks ${ }^{12}$. All samples that were positive for culture were subjected to $\mathrm{ZN}$ staining and culture on blood agar plates to confirm growth of acid fast bacilli and to rule out the contamination respectively. Confirmation of MTB from the cultures was done using MPT64 antigen test kit (SD Bioline, Korea) using manufacturer's protocol. Samples showing rifampicin resistant by GeneXpert assay were validated by performing Genotype MTBDR plus assay following manufacturer's guidelines. 
Table 1. Comparison between smear microscopy, GeneXpert and MGIT 960 culture

\begin{tabular}{lcccc}
\hline & $\begin{array}{c}\text { Smear } \\
\text { Positive }\end{array}$ & $\begin{array}{c}\text { Smear } \\
\text { Negative }\end{array}$ & $\begin{array}{l}\text { Culture } \\
\text { Positive }\end{array}$ & $\begin{array}{c}\text { Culture } \\
\text { Negative }\end{array}$ \\
\hline GeneXpert Positive & 128 & 56 & 177 & 07 \\
GeneXpert Negative & 05 & 473 & 05 & 473 \\
Sensitivity (95\% Cl) & $96.24 \%(91.44 \%-98.77 \%)$ & $97.25 \%(93.71 \%-99.10 \%)$ \\
Specificity (95\% Cl) & $89.41 \%(86.47 \%-91.90 \%)$ & $98.54 \%(97.02 \%-99.41 \%)$ \\
Positive Predictive & $69.57 \%(64.03 \%-74.59 \%)$ & $96.20 \%(92.38 \%-98.14 \%)$ \\
Value(95\% Cl) & & & \\
Negative Predictive & $98.95 \%(97.56 \%-99.55 \%)$ & $98.95 \%(97.55 \%-99.56 \%)$ \\
Value(95\% Cl) & & & \\
\hline
\end{tabular}

\section{Statistical Analysis}

Patient demographic data and all test were analysed using SPSS version 22 (SPSS Inc., USA). A $p$ value $<0.05$ was considered statistically significant for all statistical analysis.

\section{RESULTS}

A total of 662 respiratory samples comprising of sputum (211) and BAL (451) from patient suspected of tuberculosis were tested for smear microscopy and GeneXpert. The mean age \pm SD of the patients was found to be $52.7 \pm 13.56$ years (Range: $15-85$ years), 451 (68.12\%) among them were male where as $211(31.87 \%)$ were females. On comparing the results of smear microscopy and GeneXpert 128 (19.33\%) samples were found positive by both methods whereas $473(71.45 \%)$ samples were negative by both methods. Fifty six (8.45\%) samples were positive by GeneXpert alone, whereas $5(0.75 \%)$ samples were positive by smear microscopy only (Table1). When GeneXpert and smear microscopy results were compared with culture, 49 (7.40\%) smear negative but GeneXpert positive samples were found positive for culture; further $5(0.75 \%)$ smear positive but GeneXpert negative samples were also found positive for culture and were identified as Non Tuberculous Mycobacteria. Seven (1.05\%) samples were found positive alone by GeneXpert (Table 1). On comparing with culture the sensitivity, specificity, positive predictive value and negative predictive value of GeneXpert assay was found to be $97.25 \%$ (93.71\%-99.10\%), $98.54 \%$ (97.02\%-99.41\%), 96.20\% (92.38\%-98.14\%) and 98.95\% (97.55\%-99.56\%) respectively whereas sensitivity, specificity, positive predictive value and negative predictive value of smear microscopy was found to be $73.08 \%$ (66.01\%-79.37\%), $100 \%$ (99.23\%-100\%), $100 \%$ and $90.74 \%(88.52 \%-$ $92.56 \%)$ respectively. Rifampicin resistance was detected in 11 (1.66\%) samples by GeneXpert, Five $(0.75 \%)$ samples among them were found to be MDR whereas one sample showed false positive result for rifampicin resistant when compared by Genotype MTBDR plus assay.

\section{DISCUSSION}

Early detection of TB and MDR status is one of the most important factors for appropriate management of TB. In majority of resource poor countries the diagnosis of TB is based on conventional methods which are suboptimal, laborious and time consuming. Unavailability of adequate tool for rapid diagnoses of TB remains a major hindrance in achieving the global target of tuberculosis elimination. GeneXpert is a rapid automated assay for detection of TB and Rifampicin resistance directly from clinical samples. Authors in the past have reported utility of GeneXpert assay in rapid diagnosis of TB ${ }^{13-14}$. Present study was performed with an aim to evaluate the diagnostic performance of this assay for diagnosis of pulmonary tuberculosis in comparison with Auramine $O$ staining based LED-FM. The overall sensitivity, specificity, PPV and NPV of GeneXpert in present study was found to be $97.25 \%, 98.54 \%$, $96.20 \%$ and $98.95 \%$ respectively, whereas the overall sensitivity, specificity, PPV and NPV for Auramine $O$ staining based LED-FM was found to be $73.08 \%, 100 \%, 100 \%$, and $90.74 \%$ respectively which is comparable with previously published studies $^{15,8}$. A previous study from India showed 
$10.8 \%$ increment in case detection by GeneXpert when compared to fluorescent microscopy. In the present study also 49 (7.40\%) samples which were smear negative were detected positive by GeneXpert and culture ${ }^{16}$. Five smear positive samples were found negative by GeneXpert, all theses samples were later confirmed as NTM (Non tuberculous mycobacteria) by culture and Hains GenoType Mycobacterium CM assay which suggests that GeneXpert is highly specific for MTB complex. Bacterial count below detection limit of GeneXpert may also attribute to negative results ${ }^{17}$. GeneXpert showed false positive result for 7 samples, all these patients had past history of TB treatment and DNA of dead bacteria may have resulted in false positive GeneXpert result. Since rifampicin mono-resistance is rare and $90 \%$ cases of rifampicin resistance were reported to have isoniazid resistance as well, thus resistance to rifampicin is considered as a marker for MDR TB ${ }^{18}$. In present study, 5 samples out of 11 rifampicin resistant samples were found to be MDR. Authors in past has reported discrepancies in rifampicin resistance detection by GeneXpert; likewise one sample detected rifampicin resistant by GeneXpert in the present study was found to be a sensitive strain when validated on Genotype MTBDR plus ${ }^{19}$. To conclude GeneXpert offers much higher sensivity as compared to Auramine $O$ staining based LED-FM although the specificity of both is almost similar. GeneXpert assay seems to be a promising tool for TB diagnosis and has a potential to improve the case detection rates especially in smear negative cases and MDR-TB. Further longitudinal studies are required to evaluate the potential of this assay at primary health care settings and diagnosis of Extrapulmonary TB.

\section{ACKNOWLEDGEMENTS}

We would like to thank Technical Staff Miss Suraksha and Mrs Rajini for their various roles and contributions to this project.

\section{CONFLICT OF INTEREST}

The authors declares that there is no conflict of interest.

\section{AUTHORS' CONTRIBUTION}

All authors have made substantial contribution to the work and approved it for publication.

\section{FUNDING}

None.

\section{DATA AVAILABILITY}

All data generated during the study are included in the manuscript.

\section{ETHICS STATEMENT}

The study was undertaken after obtaining approval from the Institutional Research and Ethics Committee.

\section{REFERENCES}

1. World Health Organization. Global tuberculosis report 2018. Geneva, Switzerland: World Health Organization; 2018.Available at: https://apps.who. int/iris/bitstream/ handle/10665 / 274453/9789241565646-eng.pdf.

2. Van Kampen SC, Anthony RM, Klatser PR. The realistic performance achievable with mycobacterial automated culture systems in high and low prevalence settings. BMC Inf. Dis., 2010; 10(1):93. https://doi. org/10.1186/1471-2334-10-93.

3. Siddiqi K, Lambert ML, Walley J. Clinical diagnosis of smear-negative pulmonary tuberculosis in low-income countries: the current evidence. Lancet Infect. Dis., 2003; 3(5): 288-296. https://doi.org/10.1016/S14733099(03)00609-1.

4. Dorman SE. New diagnostic tests for tuberculosis: bench, bedside, and beyond. Clin. Infect. Dis., 2010; 15; 50(Supplement_3):S173-177. https://doi. org/10.1086/651488.

5. World Health Organization. Fluorescent light-emitting diode (LED) microscopy for diagnosis of tuberculosis: policy statement, 2011. Available at : http://apps.who. int/iris/bitstream/10665/44602/1/9789241501613_ eng.pdf

6. Thapa B, Reza LW, Kumar AM, Pandey A, Satyanarayana $S$, Chadha S. Light Emitting Diode Fluorescence Microscopy increased the detection of smear-positives during follow-up of Tuberculosis patients in India: program implications. BMC Res. Notes, 2015; 8(1): 596._https://doi.org/10.1186/s13104-015-1584-z.

7. Automated real-time nucleic acid amplification technology for rapid and simultaneous detection of tuberculosis and rifampicin resistance: Xpert MTB/ RIF system. Policy statement. Geneva, World Health Organization, 2011. Available at http://whqlibdoc. who.int/ publications /2011/9789241501 545_eng. pdf

8. Chang K, Lu W, Wang J, Zhang K, Jia S, Li F, et al. Rapid and effective diagnosis of tuberculosis and rifampicin resistance with Xpert MTB/RIF assay: a meta-analysis. J. Infect, 2012; 64(6): 580-588. https:// doi.org/10.1016/j.jinf.2012.02.012. 
9. Boehme CC, Nabeta P, Hillemann D, Nicol MP, Shenai S, Krapp F, et al. Rapid molecular detection of tuberculosis and rifampin resistance. N. Engl. J. Med., 2010; 363(11): 1005-1015. https://doi.org/10.1056/ NEJMoa0907847.

10. Steingart KR, Sohn H, Schiller I, Kloda LA, Boehme CC, Pai M, Dendukuri N . Xpert ${ }^{\circledR}$ MTB/RIF assay for pulmonary tuberculosis and rifampicin resistance in adults. Cochrane Database Syst. Rev., 2013; 1(1). https://doi.org/10.1002/14651858.CD009593.pub2.

11. Central TB Division. Manual of sputum smear fluorescence microscopy. Available at: http://tbcindia.nic.in/WriteReadData/ I892s/7890638455Flourescence_Microscopy\%20 Manual.pdf

12. MGITTM Procedure Manual, 2006. Available at : https://www.finddx.org/wp-content/uploads /2016/02/mgit_manual_nov2006.pdf

13. Bowles EC, Frey'e B, van Ingen J, Mulder B, Boeree MJ, et al. Xpert MTB/RIF ${ }^{\oplus}$, a novel automated polymerase chain reaction-based tool for the diagnosis of tuberculosis. Int. J. Tuberc. Lung Dis., 2011; 15: 988989. https://doi.org/10.5588/ijtld.10.0574.

14. Marlowe EM, Novak-Weekley SM, Cumpio J, Sharp SE, Momeny MA, Babst A, et al. . Evaluation of the Cepheid Xpert MTB/RIF assay for direct detection of Mycobacterium tuberculosis complex in respiratory specimens. J. Clin. Microbio., 2011; 149: 1621-1623. https://doi.org/10.1128/JCM.02214-10.
15. Sharma SK, Kohli M, Yadav RN, Chaubey J, Bhasin D, Sreenivas V, et al. Evaluating the Diagnostic Accuracy of Xpert MTB/RIF Assay in Pulmonary Tuberculosis. PLOS ONE, 2015; 10(10): e0141011._https://doi. org/10.1371/journal.pone.0141011.

16. Alvarez-Uria G, Azcona JM, Midde, M, Naik, PK, Reddy $S$, \& Reddy R. Rapid diagnosis of pulmonary and extrapulmonary tuberculosis in HIV-infected patients. Comparison of LED fluorescent microscopy and the geneXpert MTB/RIF assay in a district hospital in India. Tuberc Res. \& Treat, 2012.

17. Palud P, Cattoir V, Malbruny B, Magnier R, Campbell K, Oulkhouir $Y$, et al. Retrospective observational study of diagnostic accuracy of the Xpert ${ }^{\circledR}$ MTB/ RIF assay on fiberoptic bronchoscopy sampling for early diagnosis of smear-negative or sputum-scarce patients with suspected tuberculosis. BMC Pulm. Med., 2014; 14 (1): 137. https://doi.org/10.1186/1471-2466-14-137.

18. Drobniewski $\mathrm{F}$, Wilson $\mathrm{S}$. The rapid diagnosis of isoniazid and rifampicin resistance in Mycobacterium tuberculosis-a molecular story. J. Med. Microbiol., 1998; 47: 189-196. https://doi.org/10.1099/0022261547-3-189.

19. Rufai SB, Kumar P, Singh A, Prajapati S, Balooni V, et al. Comparison of Xpert MTB/RIF with line probe assay for detection of rifampin-monoresistant Mycobacterium tuberculosis. J. Clin. Microbiol., 2014; 52: 1846-1852. https://doi.org/10.1128/JCM.03005-13. 